\title{
Recognition of Oracular Bone Inscriptions Using Template Matching
}

\author{
Lin Meng, Yoshiyuki Fujikawa, Atsushi Ochiai, Tomonori Izumi, and Katsuhiro Yamazaki
}

\begin{abstract}
Although several inscription recognition methods have been proposed, the experiments are not enough. We propose a novel approach to recognize the inscriptions by template matching. The techniques include Gaussian filtering, binarization, labeling, thinning, Hough transform and template matching. In order to reduce noises, we propose a four-directional scan labeling. The target inscriptions are selected randomly from the scanned inscriptions rubbing of book. The template matching compares the rubbing inscriptions with normalized inscriptions which are selected from an inscriptions database. The normalized inscriptions are generated by character font software, which makes the characters smooth, clear, uniformly thick strokes, and straight. The experiment results show that $87 \%$ of 31 inscriptions are correctly recognized.
\end{abstract}

Index Terms-Oracular bone inscription, recognition, labeling, template matching.

\section{INTRODUCTION}

Oracular bone inscriptions are one of the oldest characters in the world, which are inscribed on bone of cattle, turtle shells etc. about 3000 years ago [1]. Reading these oracular bone inscriptions makes it possible to get an understanding of the pre Christ era. However, aging caused these inscriptions to be less legible.

This paper describes how to recognize the oracular bone inscriptions by using template matching which compares the line extracted inscriptions with a template in the inscriptions database. The line extracted inscriptions are generated from the scanned rubbing image of oracular bone inscriptions.

The process uses the Gaussian filtering and labeling to reduce the noises of rubbing inscriptions image, and employs thinning and Hough transform to extract inscriptions lines. Then the extracted lines of inscriptions are compared with a template in order to recognize the inscriptions.

Since labels are generated by only referring the scanned neighboring pixels in usual labeling, the labeling to be erroneous in some occasions. We propose a four-directional scan labeling to scan the image from four directions and select the least label count direction as the final accurate label.

In the template matching, the line extracted inscriptions

Manuscript received May 19, 2014; revised November 16, 2014.

Lin Meng, Yoshiyuki Fujikawa, Tomonori Izumi, and Katsuhiro Yamazaki are with the Department of Electronic and Computer Engineering, Ritsumeikan University, Kusatsu, Shiga, Japan (e-mail: \{menglin@fc, fujikawa@hpc.se, t-izumi@se, yamazaki@se\}.ritsumei.ac.jp).

Atsushi Ochiai is with the College of Letters, Ritsumeikan University, Kusatsu, Kyoto, Japan (e-mail: aot23191@1t). are compared with the templates which were developed at Ritsumeikan University.

In the experiments, the input oracular bone inscription rubbing, were selected randomly from book [2], and the inscription templates were selected from the inscriptions database [3].

The contributions of this paper are as follows:

- Develops a recognition method for recognizing the oracular bone inscriptions by using template matching.

- Proposes a four-directional scan labeling to reduce the noises and generate the correct labels.

The rest of this paper is organized as follows. Section II explains related work. Section III describes the recognition method. Section IV reports the four-directional scan labeling. Experiments and conclusion are stated in Section V and Section VI.

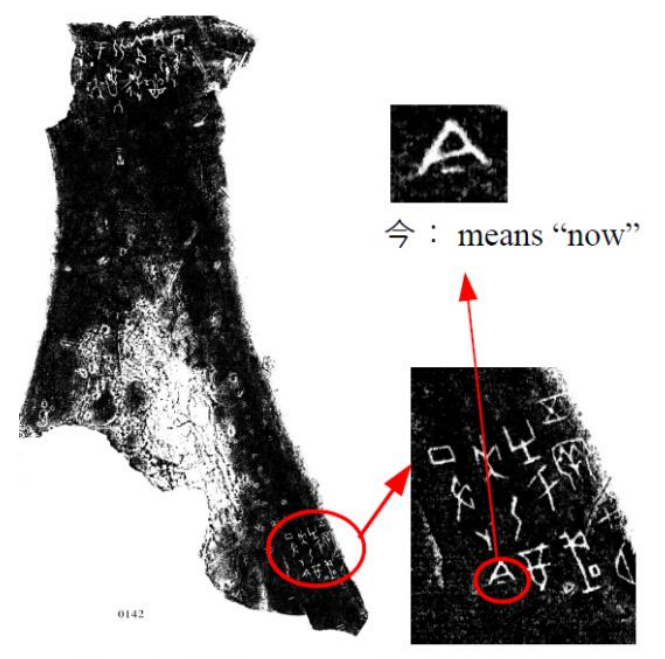

$\begin{array}{ll}\text { (a) A rubbing } & \text { (b) An expanded edition }\end{array}$ Fig. 1. An oracular bone inscription.

\section{RELATED WORK}

\section{A. Oracular Bone Inscripthions}

The oracular bones were first found in 1899, and have been buried in ruins for about 3000 years. The aging caused the inscriptions to be illegible.

Fig. 1 shows an image of oracular bone inscriptions. Fig. 1(a) is a rubbing and Fig. 1(b) is zoomed portion of the rubbing.

\section{B. Recoginition of Oracular Bone Inscriptions}

Presently, historians read oracular inscriptions by referring to the context of existing oracular inscriptions.

$\mathrm{Li}$ and Woo proposed a coding principle and method to recognizing such inscriptions [4]. This method treats the inscriptions as a non-directed graph by detecting the 
end-points, cross-points, blocks and net-holes.

Li and Yang proposed a sticker DNA algorithm and designed a DNA coding method suited to DNA computer for recognizing the inscriptions [5].

$\mathrm{Li}$, Yang and Wang proposed an algorithm based on graph isomorphism [6]. In this method, inscriptions are transformed to the labeled graphs, and the inscriptions are encoded by the quasi-adjacency matrix of the labeled graphs.

We propose not only a four-directional scan labeling to reduce noises, but also a novel recognition method by using template matching. The template matching compares the rubbing inscriptions with normalized inscriptions which are selected from an inscriptions database.

\section{RECOGNITION OF ORACULAR BONE INSCRIPTIONS}

\section{A. Overview of Oracular Bone Inscription Recognition}

Fig. 2 represents the recognition flowchart. The inscriptions are input by an image scan of rubbing from book [2] which keeps more than 5000 pieces of oracular bones. The inscriptions database keeps the normalized inscriptions images similar to a dictionary. The normalized inscriptions are generated by character font software, which makes the characters smooth, clear, uniformly thick strokes, and straight. These characters have been read by historians, and have been stored as a normalized inscriptions database by the Letters College of Ritsumeikan University. The database keeps more than 2000 normalized inscriptions.

The recognition process is done as follows: Gaussian filtering reduces the smaller noises before the binarization. Labeling reduces the larger noises. Thinning extracts the frames of the inscription image. Hough transform extracts the lines from the inscription frame. Template matching compares the Hough transform result with templates to recognize the inscriptions.

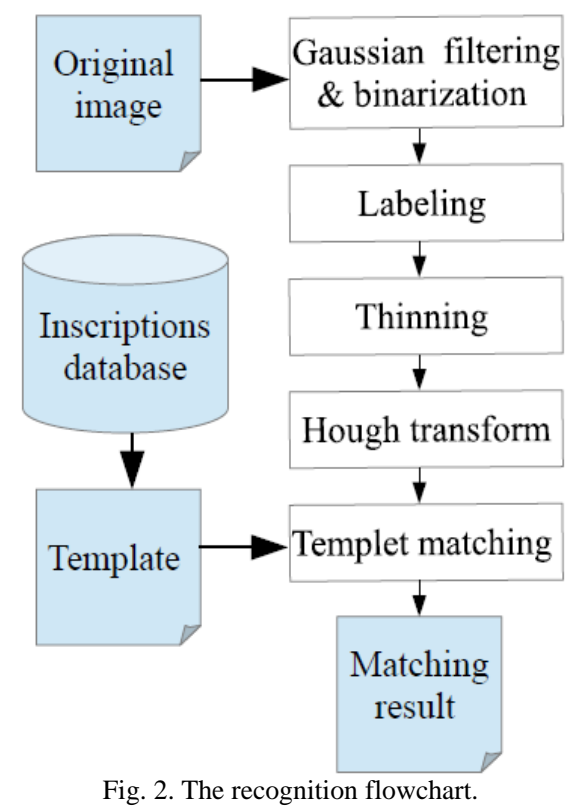

\section{B. Image Processing}

Here, we use “貞” to explain the recognition process. Fig. 3 (a) is an original inscriptions image.

\section{1) Gaussian filtering and binarization}

Gaussian Filtering is used to blur image and remove noises by using the Gaussian function. Binarization changes each pixel of the above digital image to either value 255 or 0 by comparing with a threshold value.

Fig. 3(b) shows the Gaussian Filtering and binary image, which becomes clearer than the original image after the process. However there are many larger dots in the right bottom of the image as the noises.

\section{2) Labeling}

The usual labeling gives the same object a same label number [7]. It consists of temporary label generation and revising labels.

Temporary label generation scans the four-neighbor (upper left, up, upper right, and left) of the target pixel after the binarization stage. If the target pixel is 255 , a temporary label is generated. When four neighbors have label numbers, the label is copied in the following order of priority: upper left, up, upper right, and left. Otherwise, a new label number is generated.

Revising Labels (RLabeling) is used to revise the temporary labels. Here, the labels of the eight pixels neighboring the target pixel is compared with each other, and the lowest numbered label is used as the label of the target pixel when many target labels exist.

By using the usual labeling, it sometimes makes mistakes. We proposed a four-directional scan labeling to correct this mistake. In section IV we will explain the labeling method in detail. Fig. 3(c) shows the proposed labeling result which deleted the noises.

\section{3) Thinning}

Thinning is conducted to extract the frames of the inscription image. We used Tamura method for this task This method prepares the eight-neighbor patterns for thinning. The thinning function scans from the top of the image, and when the eight-neighbor are matching with one of the eight-neighbor patterns, the target pixel will be reversed. Results of thinning is shown in Fig. 3(d).
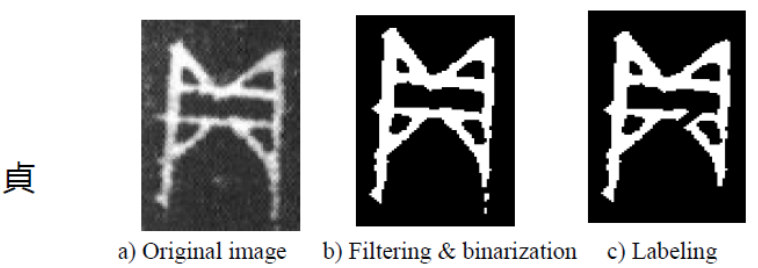

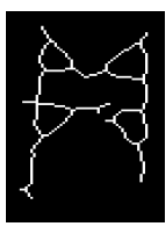

d) Thinning

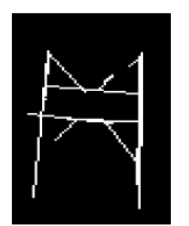

e) Hough Tranform

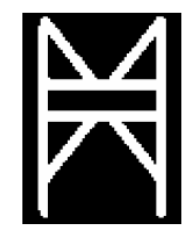

f) Template

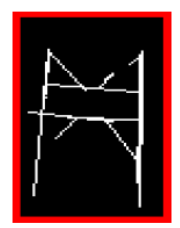

g) Matching result
Fig. 3. An example of the matching process.

\section{4) Hough transform}

Hough Transform uses equation (1) to extract the direct line. $x, y$ are the axes of image, $\rho$ represents the distance between the line and origin, $\theta$ is the angle of the vector from the origin to the closest point. From equation (1) the $x$, 
$y$ coordinates are transformed to $\rho, \theta$ coordinates. Next, the number of lines that share the same coordinates are noted. The most lines that share the same coordinate is selected as a line in the image. Hough transform result is shown in Fig. 3(e).

$$
\rho=x^{*} \cos \theta+y^{*} \sin \theta
$$

\section{5) Template matching}

Template Matching is a method to compare two images to search for a common area. Here, our research uses NCC (Normalized Correlation Coefficient) expressed in equation (2) to decide if the target image matches with templates [8].

$$
R(i, j)=\frac{\sum_{J=0}^{N-1} \sum_{i=0}^{M-1} I(i, j) T(i, j)}{\sqrt{\sum_{j=0}^{N-1} \sum_{i=0}^{M-1} I(i, j)^{2} * \sum_{j=0}^{N-1} \sum_{i=0}^{M-1} T(i, j)^{2}}}
$$

$i, j$ are the axis of image, $M, N$ are the maximum of axis size. $I$ is line extracted image like Fig. 3(e) which is generated from a copied really rubbing, $T$ is template image like Fig. 3(f) which is copied form normalized inscriptions database. $R$ is the NCC result of two images. When the result is closer to 1 , the similarity is higher. Template matching result is shown in Fig. 3(g), the matched area is surrounded by red box.

\section{FOUR-DRECTIONS SCAN LABELING}

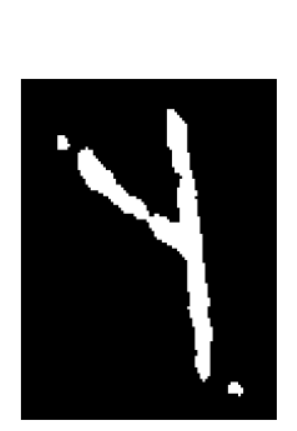

a) Binarization image

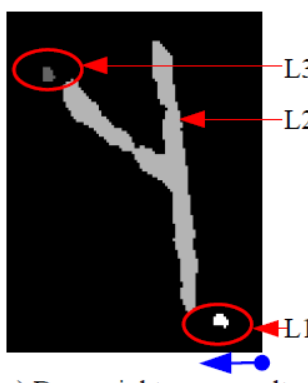

c) Down right scan result d) The detected label

Fig. 4. An example to depict the process of labeling.

In labeling, we give each object a label number and count the pixels of the object. When the label numbers are too small, we ignore the labels as noises.

The usual labeling method often makes errors because the labels are generated by only referring the scanned neighbor.
For example, Fig. 4(b) presents the results of a usual labeing of the binary image of Fig. 4(a). When the first pixel of Label 3 (L3) is scanned, there is no scanned labels on the neighboring pixels. Therefore, L3 is created as a new label, even if it was connected with L1.

In this work we propose a four-directional scan labeling to generate the labels. Here, the scanning direction that has the least number of labels is used as the final label. The scanning starts from Top left (TL), Top right (TR), Down left (DL), Down right (DR).

Fig. 4 (b) starts from TL and Fig. 4(c) starts from DR. The DR has the least number of labels. Thus the final labeling uses the DR result.

To reduce the noises we recoded the pixels of individual labels, and calculated the ratio of pixels in each label. Nex we ignored the labels which had the least numbers of pixels.

\begin{tabular}{|c|c|c|}
\hline \multicolumn{3}{|c|}{ TABLE I: RATIO OF LABEL PIXELS } \\
\hline Label Number & Number of Pixels & Ratio of pixels (\%) \\
\hline 1 & 22 & 2.2 \\
\hline 2 & 991 & 95.8 \\
\hline 3 & 21 & 2.0 \\
\hline
\end{tabular}

Table I lists the ratio of pixels in labels. Label 1 and label 2 only occupy $2 \%$ of the object's pixels, making them to be considered as noises. The detected label is shown in Fig. 4(d), it could be observed that the two dots in the Fig. 4(a) are eliminated as noises.

\section{EXPERIMENTATION AND DISCUSSIONS}

\section{A. Experimental Conditions}

The CPU uses an Intel ${ }^{\circledR}$ Core $^{\mathrm{TM}} \mathrm{i} 7-3820$ Processor with a clock speed of $3.60 \mathrm{GHz}$ and $8.00 \mathrm{~GB}$ onboard memory. Ubuntu 13.10 is used as the operating system. C and OpenCV libraries are used as the programming language. GCC 4.8 is the compiler, and the version of OpenCV is 2.4.9.

The image processing steps namely: Gaussian filtering, binarization, Hough transform, thinning, and template matching are conducted using OpenCV library functions. Labeling is conducted by personally written $\mathrm{C}$ code.

The 31 target inscriptions are scanned randomly from book [2], and are compared with the corresponding templates selected from database [3].

\section{B. Experimetion Results}

Fig. 5 and 6 present simple and complex characters recognized correctly by the proposed method.

From Fig. 5(b) and 6(b), it could be noted that in the processed image, the smaller noises are reduced and made clearer than the original image. However there are few dots present as they are comparatively larger noises. Fig. 5(c) and 6(c) depicts that the larger noises were reduced when four directional scanning is used for labeling.

Fig. 5(d, e), and Fig. 6(d, e) show images obtained after the Thinning and Hough Transformation are conducted. The lines are abstracted in these images.

Fig. 5(g), Fig. $6(\mathrm{~g})$ are matching results which are compared with line abstracted image of Fig. 5(e), Fig. 6(e) and the templates of Fig. 5(f), Fig. 6(f). The read lines mark 
the matched area.

The method recognizes the following inscriptions (besides Fig. 5 and Fig. 6) successfully: 己、用、矢、死、 丘、受、宜、月、方、禍、貞、今、観、省、争、崇、 茲、王、卜

The following inscriptions could not be identified: 衛、黍、 勿、酒.
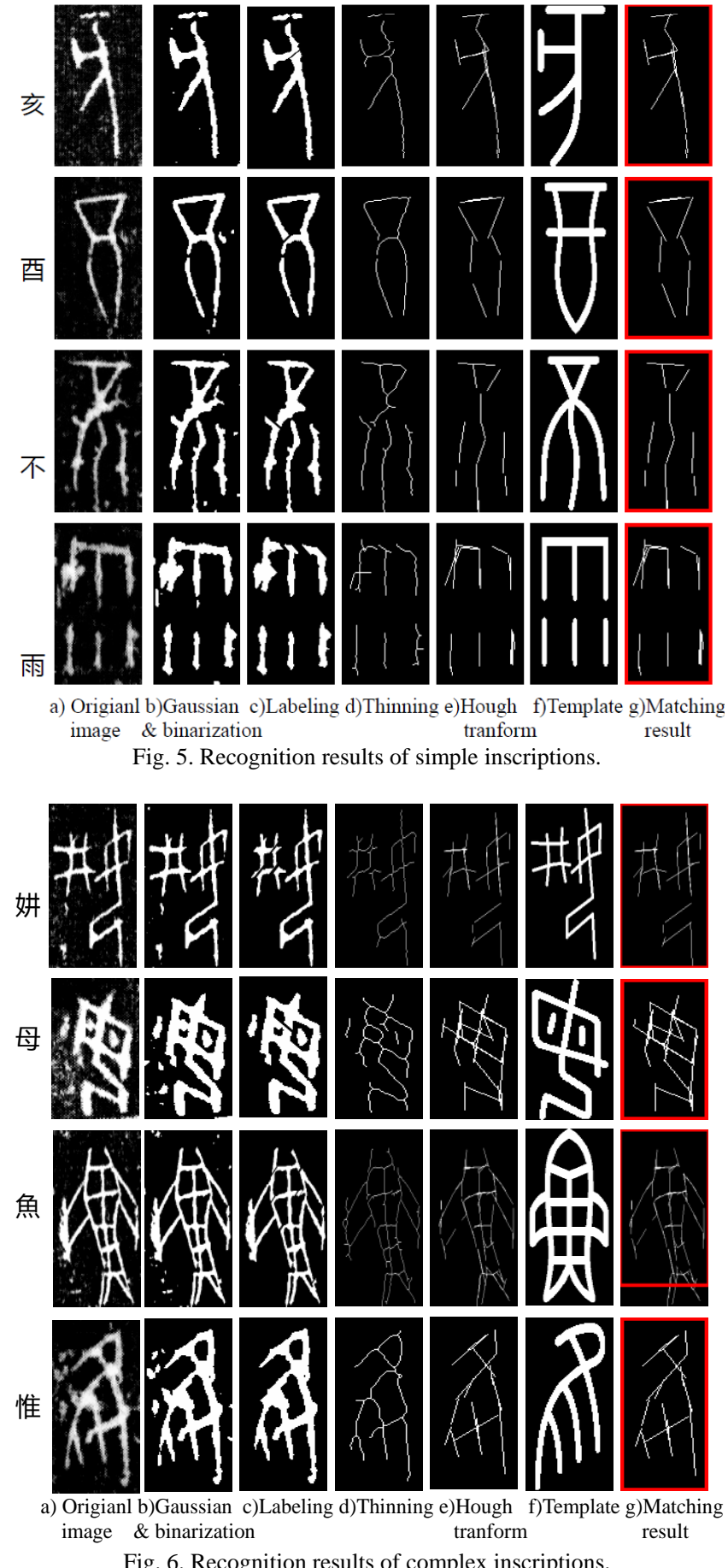

\section{Disscussion}

The proposed method recognizes inscriptions with a high accuracy. It could be noted that between Gaussian filtering and labeling, he noises of the image was reduces significantly. Thinning extracts the frame, while Hough transform extract the line of the characters. However, some noises such as mustachios exists in the Hough transformed

result. Researching ways to reduce these mustachios noises is considered in the future.

Currently, we have only compared the template with the 31 random selected inscriptions with the similar template. In future, we will attempt to search the target inscriptions lined images in inscriptions database, automate the recognition of the inscriptions.

\section{CONCLUSION}

This paper has described how to recognize oracular bone inscriptions by using template matching which compares the copied rubbings with a template in the inscriptions database. In addition, we have proposed a four-directional scan labeling.

The experiments show that our method achieves about $87 \%$ recognition rate by comparing the rubbing of inscriptions with the normalized inscription templates. The four-directional scan labeling generated correct labels by reducing the noises of the inscription image.

Future issues are testing more inscriptions and attempting to search candidate templates from the inscriptions database for recognizing inscriptions automatically.

\section{ACKNOWLEDGMENT}

This work was supported in part by a grant-in-aid for scientific research (26870713) from JSPS.

\section{REFERENCES}

[1] A. Ochiai, Reading History from Oracular Bone Inscriptions, Chikuma Shobo, 2008

[2] M. Z. Pu and H. Y. Xie, Shanghai bo wu guan cang jia gu wen zi, Shanghai Bo Wu Guan, 2009.

[3] A. Ochiai. [Online]. Available: http://koukotsu.sakura.ne.jp/top.html

[4] F. Li and P. Y. Woo, "The coding principle and method for automatic recognition of Jia Gu Wen characters," International Journal of Human-Computer Studies, vol. 53, pp. 289-299, 2000.

[5] Q. S. Li and Y. X. Yang, "Sticker DNA algorithm of oracle-bone inscriptions retrieving," Computer Engineering and Applications, vol. 44, no. 28, pp. 140-142, 2008.

[6] Q. S. Li, Y. X. Yang, and A. M. Wang, "Recognition of inscription on bones or tortoise shells based on graph isomorphism," Computer Engineering and Applications, vol. 47, no. 8, pp. 112-114, 2011

[7] L. F. He, Y. Y. Chao, K. J. Szuki, T. Nakamura, and H. Itoh, "An efficient two-scan connected-component labeling algorithm," IEICE Transaction on Information and Systems, vol. J91-D, no. 4, pp 1016-1024, 2008.

[8] T. Mahalakshimi, R. Muthiaiah, and P. Swaminathan, "Review Article: An overview of template matching technique in image processing," Research Journal of Applied Sciences, Engineering and Technology, vol. 4, no. 24, pp. 5469-5473, 2012.
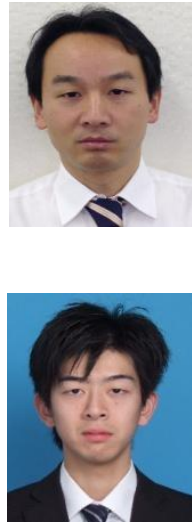

Lin Meng is an assistant professor at the Department of Electronic and Computer Engineering, College of Science and Engineering, Ritsumeikan University in Japan. His research interests include computer architecture and image processing. He received a Ph.D degree in computer science from Ritsumeikan University in 2012. He is a member of IEICE and IPSJ.

Yoshiyuki Fujikawa is a master student at the Department of Electronic and Computer Engineering, Ritsumeikan University in Japan. His research interests are in the area of image processing about oracular bone inscription recognition. He received his B.E. degree in the Department of VLSI System Design, Ritsumeikan University. He is a student member of IPSJ. 


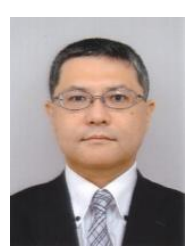

Atsushi Ochiai is a visiting researcher at Shizuka Shirakawa Memorial Oriental Character Culture Laboratory, Ritsumeikan University in Japan. His research interests include ancient history of China and inscriptions on oracle bones. He received a Ph.D. degree in literature from Ritsumeikan University in 2001. He is a member of The Society of Historical Research and The Toho Gakkai.

Tomonori Izumi is an associate professor at Department of Electronic and Computer Engineering, Ritsumeikan University, Japan. His research interests include system, design and design methodologies of reconfigurable hardware. He received his Ph.D. degree in electrical and electronic engineering from Tokyo Institute of Technology in 1998. He is a member of IEEE, IEICE, and IPSJ.

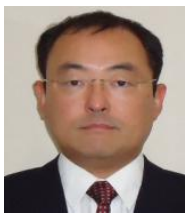

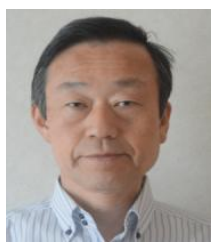

Katsuhiro Yamazaki is a professor at the Department of Electronic and Computer Engineering, College of Science and Engineering, Ritsumeikan University in Japan. His research interests include parallel computing, and hardware/software codesign. He received a Ph.D. degree in computer science from Kyoto University in 1986 . He is a member of IEICE, IPSJ, ACM and IEEE. 